\title{
Design and implementation of fingerprint access control system based on ZigBee wireless network
}

\author{
Jin Zhang ${ }^{1}$,Shaoyong Jian ${ }^{*}$ \\ ${ }^{1}$ Changzhou Vocational Institute of Engineering.Changzhou,213000,China \\ *Department of Mathematics and Computer Sciences, Xinyu University, Xinyu,338004,China
}

\begin{abstract}
: this paper mainly introduces a kind of fingerprint access control system based on ZigBee wireless network design and implementation method, specify when passengers should fingerprints collected and stored in the system at the information desk PC, and then assign rooms; Guests entered the room according to the fingerprint module, the system will be fingerprint information through ZigBee coordinator node module and network to transmit the fingerprint characteristic value to management system and the fingerprint information stored, if consistent with the electromagnetic lock open, allowed to enter the room, does not conform to the issued a warning sound.Additional access control system can control the corresponding fingerprint information storage room and remove, such as is required for a security incident broke in situation is special open mode can be set up.System software design consists of two aspects of the bottom and upper machine.
\end{abstract}

\section{The introduction}

Hotels are a place where people stay short and change quickly, which brings convenience and safety hazards to the people who provide them. Often in the news, the suspect in the news of the suspect in the fake hotel guest to open the door of the room to steal, although there are surveillance equipment and see the suspect, but solving the case is not easy. Even if the amount of loss is small, but some id CARDS, credit CARDS and other things, it is troublesome to apply for a replacement.

Hotels access control system from the original key to the current popular induction card, the goal of the implementation is to be safe, convenient. However, in these systems, there is a fundamentally unsolvable problem where the key can be copied, the sensor card can be stolen, or the user identity cannot be identified. Therefore, the ultimate goal of the development of the gated system can only be biometric identification. At present, the common biometric identification system has fingerprint, iris, etc. Relatively, the fingerprint identification is simpler, and there is more choice. Therefore, if the hotel and hotel access control system is changed to the fingerprint access system, the thief will have nothing to do and greatly improve the security of housing.

But one problem that needs to be addressed is how to 
design the minimum cost of access control system transformation program. The network transmission scheme of fingerprint eigenvalue information is usually transmitted by GPRS, Wifi, ZigBee and other modes. Look from the actual application effect, the wireless mode sensing module installation is simple, the equipment maintenance workload small, the advantages of the engineering costs are relatively cheap makes it have a huge impact to the traditional cable transmission mode.

\section{System structure and design thinking}

Fingerprint access control system based on ZigBee wireless network is divided into fingerprint input module and fingerprinting module, fingerprint input module is mainly to collect the fingerprint characteristic value of passenger and entry to PC system, interface designed by QT. Fingerprinting module mainly by the fingerprint acquisition module, ZigBee terminal module and ZigBee coordinator of the fingerprint characteristic value of the package and sent to ZigBee terminal, through the ZigBee real-time calibration coordinator and the upper machine, than success, return to unlock signal, open the electromagnetic lock. If the fingerprint is not successful, the fingerprint information will be collected. If the fingerprint information is not successful, the alarm signal will be generated and the access control function will be realized.

The design idea mainly considers in the following aspects:

\subsection{Biometric identification to prevent counterfeiting;}

When checking in, the guest needs to register the fingerprint information and store it on the system's upper computer. The terminal will collect the fingerprint information and compare it with the upper computer via the ZigBee wireless network. The match will open the electromagnetic lock, otherwise the error will be prompted, and a number of errors will generate an alarm will not be opened again. signal. After the passenger has checked out, the fingerprint information will be deleted and the door lock
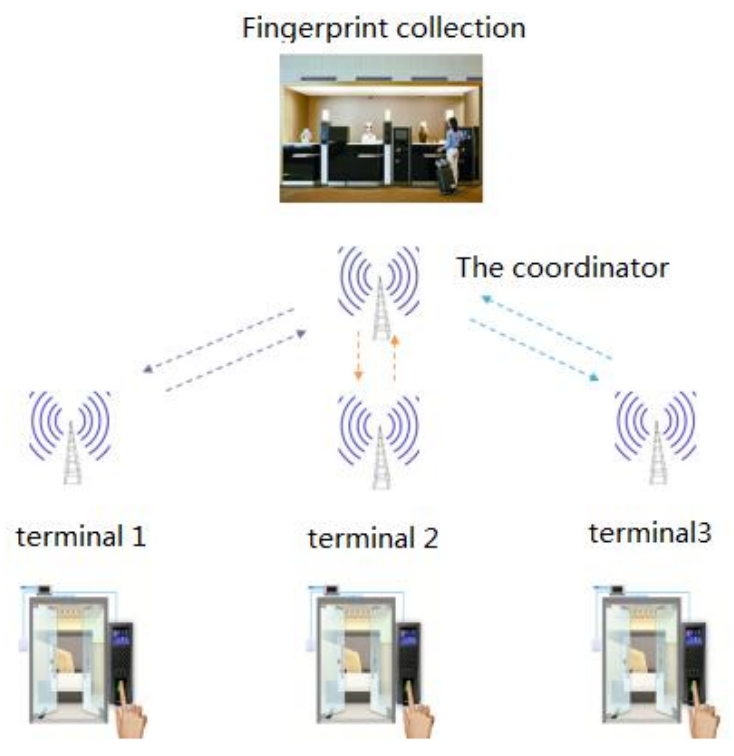

FIG. 1 schematic diagram of the system structure

2.2 Can quickly add authorized person, delete authorized person, record user and other operations;

2.3 Based on ZigBee's wireless network, the fingerprint information can be transmitted safely without upgrading the existing network.

Fingerprint information needs to be sent to the server for validation, using traditional lines of cable transmission will produce a great burden, so first consider adopting wireless fingerprint characteristic value in the form of transfer.

Despite the selection of various wireless networks, the ZigBee wireless sensor network has considerable advantages over the GPRS and other communications networks and WiFi wireless networks. The use of wireless networks such as GPRS or CDMA should be considered as the first consideration of the cost. Combined with the monthly fee, it will be difficult to accept. The advantage of WiFi is that the technology r\&d threshold is low and the product cost is low. But the biggest problem is low security and poor wireless stability. Besides, the power consumption of WiFi is also very weak, and the number of devices that the 
application system can connect is very limited, so the future development space is limited.

ZigBee technology has the advantages of high security, low power consumption and strong networking ability, compared with ZigBee wireless transmission fingerprint characteristic value.

\section{System hardware design}

The main components of ZigBee's wireless fingerprint access terminal are divided into three main components:

\subsection{CC2530 core board}

The separation of the core plate and the function board is more convenient for the maintenance and testing of the equipment. It has 21 universal $\mathrm{I} / \mathrm{O}$ pins and two $20 \mathrm{~mA}$ current absorbing or current supply capacity. The working band is $2.4 \mathrm{gHZ}$. Low power consumption, receiving current $<30 \mathrm{~mA}$, emission current $<50 \mathrm{~mA}$. Use 2. $4 \mathrm{G}$ full - to antenna, reliable transmission distance of 250 meters. Automatic reconnect is as high as 110 meters.

\subsection{ZigBee node module.}

The ZigBee node module has a size of $15^{*} 10 \mathrm{~cm}$, and its power supply mode is square port USB, DC2. 1 power base $(5 \mathrm{~V})$. Serial port communication is performed by using the USB transfer serial port function (pl-2303). With the Debug interface, it is compatible with TI standard simulation tool, which brings out all IO ports, commonly used serial port pins and $5 \mathrm{~V} / 3.3 \mathrm{~V}$ pins and other functional interfaces.

\subsection{TFS-M711 fingerprint module.}

TFS-M711 and ZigBee node module and CC2530 core board are connected by serial port and finally communicate with the upper computer.

\subsection{Overall structure of system software}

ZigBee wireless fingerprint access control system software development mainly divided into two areas: one is in the PC QT management control interface input fingerprint information, room number and distribution, at the same time save the fingerprint information used for comparison. The second is to collect the fingerprint information on the fingerprint module of the guest room, and send it to the management system by the ZigBee module and the coordinator to check the fingerprint information and return the judgment result to the guest room. Use the lock correctly, otherwise the alarm signal will be generated. The details are shown below:

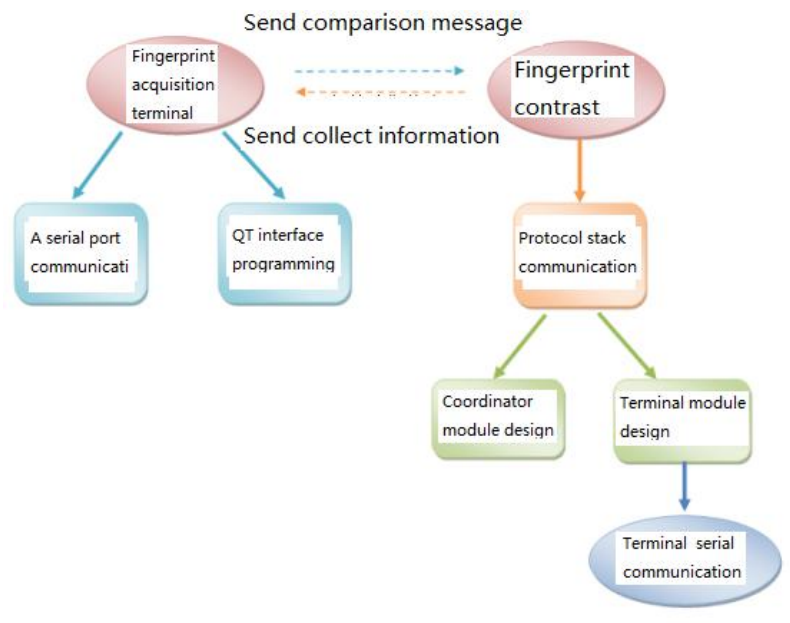

FIG. 2 overall architecture of the software

\subsection{Upper computer software design}

The upper computer software is divided into two modules: QT serial port design and QT interface design respectively. The serial port design module of QT mainly includes the serial port design of the fingerprint input module, the serial port design of the ZigBee coordinator and the sending and receiving design of the serial port. The interface design of QT mainly includes graphical module design of interface, signal slot design of interface and initializing design of constructors.

\section{System software design}




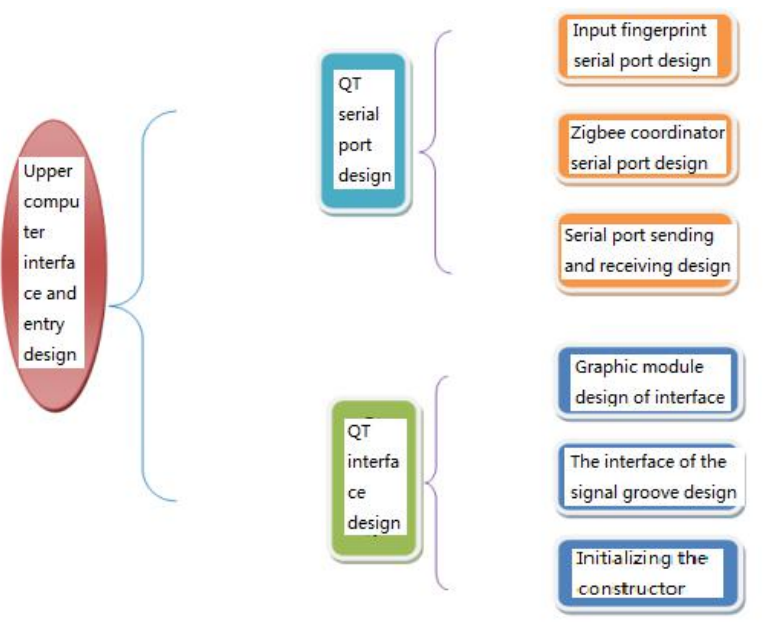

FIG. 3 upper computer programming structure

User input interface design is shown as follows:

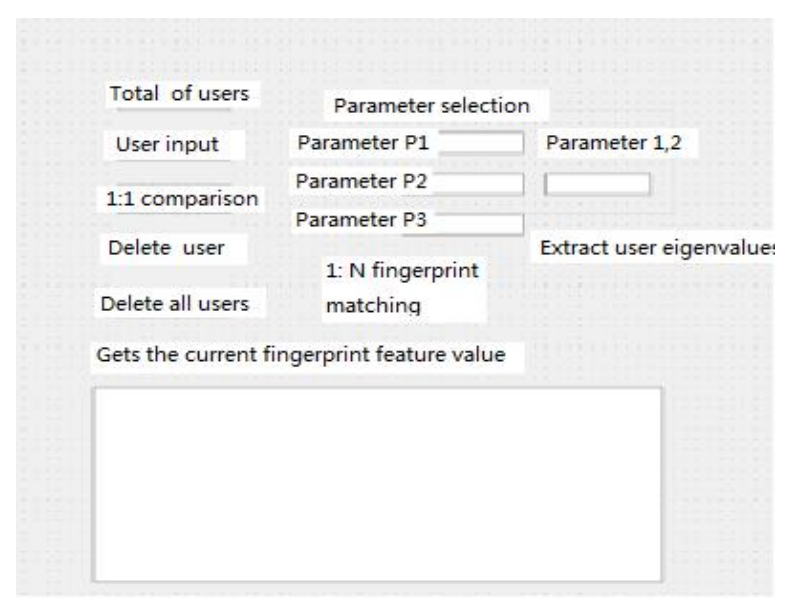

FIG. 4 user input QT interface design

\section{The conclusion}

The tenant can no longer worry about room card theft, or falsely use another identity into the room, his fingerprint is the key to the room, at the same time of convenience for passengers, further improve the performance of the hotel safe.

\subsection{Centralized management control of the reception desk.}

When checked in at the customer service desk management computer can directly input fingerprint information entry, when the check-out is removed from the entrance guard management system fingerprint information of the customers, and be able to access to any fingerprints for remote control. At the same time, the system can record the opening time of the fingerprint and send it to the front desk management system, and it can generate customer billing information and make it easier to manage.

\subsection{The supervision room authorizes manual intervention function.}

When some special events, such as: fire, kidnapping, catch the thief, etc., the monitoring center can be directly authorized to control the switch of access control points, so that the flexible handle emergency events, ensure users get more time and reduce the loss, etc.

Because the identity information, the corresponding relationship between the fingerprint of the fake id hotel also has a huge deterrent effect, has a poor record of customer if identity information does not accord with the fingerprint will automatically generate alarm signal.

\section{References:}

1. Guo xie chao, "information security" (2009.1), based on ZigBee network's wireless fingerprint identification system

2. Pan wei, "science and technology and innovation", based on ZigBee network's wireless fingerprint identification system design and implementation

3. Network bee, ZigBee combat drills v3.1 experimental manual

4. Wang xiaoqiang, ouyang jun. Design and implementation of ZigBee wireless sensor network, chemical industry press

5. Lu wenzhou, Qt5 development and examples (2nd edition), electronic industry press 